\title{
Medo, ansiedade e odontalgia em pacientes atendidos em uma Clínica-Escola de Odontologia
}

Fear, anxiety, and toothache in patients assisted at a clinic school of Dentistry Miedo, ansiedad y odontalgia en pacientes asistidos en una clínica escolar de Odontología

Maria Luísa de Assis BRAGA ${ }^{\mathbf{1}}$ Aryadna Kelley Luz ALMEIDA ${ }^{2}$

Renata Andrea Salvitti de Sá ROCHA ${ }^{3}$ Luciana Ellen Dantas COSTA ${ }^{3}$

Faldryene de Sousa QUEIROZ ${ }^{3}$

${ }^{1}$ Graduanda em Odontologia na UFCG - Centro de Saúde e Tecnologia Rural, Universidade Federal de Campina Grande (UFCG), 58708-110 Patos, PB, Brasil

${ }^{2}$ Graduada em Odontologia na UFCG - Centro de Saúde e Tecnologia Rural, Universidade Federal de Campina Grande (UFCG), 58708-110 Patos, PB, Brasil

${ }^{3}$ Professora Doutora do Curso de Odontologia na UFCG - Centro de Saúde e Tecnologia Rural, Universidade Federal de Campina Grande (UFCG), 58708-110 Patos, PB, Brasil

\section{Resumo}

Introdução: O medo, a ansiedade e a odontalgia ("dor de dente") interferem diretamente nos tratamentos odontológicos. Objetivo: Avaliar o perfil dos usuários e a prevalência e intensidade da odontalgia, ansiedade e medo relacionado ao tratamento odontológico de pacientes que procuraram atendimento na Clínica-escola de Odontologia da Universidade Federal de Campina Grande - UFCG. Metodologia: Os dados foram coletados no período de Julho a Setembro de 2018, por meio de entrevista direta aos pacientes, através de formulários semiestruturados e específicos: o Dental Fear Survey, Avaliação da Dor de Dente e Modified Dental Anxiety Scale. Os dados foram submetidos ao teste do Qui-quadrado, com nível de significância de $5 \%$. Resultados: Foram entrevistados 187 pacientes, sendo $71,1 \%$ do sexo feminino, 47,1\% pardos e com idade média de 49,1 anos. Pôde-se observar que $85 \%$ dos pacientes já sentiram "dor de dente" na vida, classificada como "Intolerante" para a maioria e 32,1\% sentiram dor nos últimos 6 meses. A maioria dos pacientes relataram não sentir ansiedade e medo frente ao tratamento odontológico. A odontalgia apresentou relação estatisticamente significante com a raça $(p=0,016)$, enquanto a ansiedade com o sexo $(p=0,022)$ e a raça $(p=0,013)$. Conclusão: Constatou-se uma baixa prevalência de medo e ansiedade e alta prevalência de odontalgia. O sexo feminino e a raça branca apresentaram maiores prevalências de ansiedade ao tratamento.

Descritores: Odontalgia; Ansiedade; Medo; Odontologia.

\section{Abstract}

Introduction: Fear, anxiety and toothache directly interfere with dental treatments. Objective: To evaluate the profile of users and the prevalence and intensity of toothache, anxiety and fear related to the dental treatment of patients who sought care at the Dental School-Clinic of the Federal University of Campina Grande - UFCG. Methodology: Data were collected from July to September 2018, through direct interviews with patients, using semi-structured and specific forms: the Dental Fear Survey, Toothache Assessment and Modified Dental Anxiety Scale. The data were submitted to the Chi-square test, with a significance level of $5 \%$. Results: 187 patients were interviewed, $71.1 \%$ were female, $47.1 \%$ were brown and had an average age of 49.1 years. It was observed that $85 \%$ of patients have already experienced toothache in their lives, classified as "Intolerant" for the majority and $32.1 \%$ have experienced pain in the last 6 months. Most patients reported not feeling anxiety and fear when facing dental treatment. Toothache showed a statistically significant relationship with race $(p=0.016)$, while anxiety with sex $(p=$ $0.022)$ and race $(p=0.013)$. Conclusion: There was a low prevalence of fear and anxiety and a high prevalence of toothache. The female sex and the white race had a higher prevalence of treatment anxiety.

Descriptors: Toothache; Anxiety; Fear; Dentistry.

Resumen

Introducción: El miedo, la ansiedad y el dolor de muelas interfieren directamente con los tratamientos dentales. Objetivo: evaluar el perfil de los usuarios y la prevalencia e intensidad del dolor de muelas, la ansiedad y el miedo relacionados con el tratamiento dental de los pacientes que buscaron atención en la Clínica-Escuela Dental de la Universidad Federal de Campina Grande - UFCG. Metodología: Los datos se obtuvieron de julio a septiembre de 2018, a través de entrevistas directas con pacientes, utilizando formas semiestructuradas y específicas: la Encuesta de miedo dental, la Evaluación del dolor de muelas y la Escala de ansiedad dental modificada. Los datos se sometieron a la prueba de Chi-cuadrado, con un nivel de significación del 5\%. Resultados: Se entrevistaron 187 pacientes, siendo $71.1 \%$ mujeres, $47.1 \%$ marrones y con una edad promedio de 49.1 años. Se observó que el $85 \%$ de los pacientes ya habían experimentado dolor de muelas en la vida, clasificados como "intolerantes" para la mayoría y el 32,1\% habían experimentado dolor en los últimos 6 meses. La mayoría de los pacientes pacientes manifestaron no sentir ansiedad y miedo al enfrentarse al tratamiento dental. El dolor de muelas mostró una relación estadísticamente significativa con la raza $(p=0.016)$, mientras que la ansiedad con el sexo $(p=0.022)$ y la raza $(p=0.013)$. Conclusión: Hubo una baja prevalencia de miedo y ansiedad y una alta prevalencia de dolor de muelas. El sexo femenino y la raza blanca tuvieron una mayor prevalencia de ansiedad por el tratamiento.

Descriptores: Odontalgia; Ansiedad; Miedo; Odontología.

INTRODUÇÃO

A dor e a ansiedade exercem significativo impacto negativo na qualidade de vida dos pacientes ${ }^{1}$. Diferentes fatores têm sido associados à presença e percepção da dor dentária, tais como: piores condições socioeconômicas, presença de carie dentária, dificuldades relacionadas à alimentação e distúrbios do sono².

Para Felix et al. ${ }^{3}$ o nível de dor está relacionado com a ansiedade, de tal modo que quanto maior for a ansiedade, maior será a dor do indivíduo. Medo e ansiedade são sentimentos comuns a pacientes que necessitam de tratamento odontológico, representando uma barreira ao atendimento adequado do profissional ou mesmo uma das causas da não procura dos serviços de saúde, justificando o distanciamento dessa população 
na busca por procedimentos preventivos ${ }^{4,5}$.

O medo pode ser definido como o temor a algo ou alguma coisa que represente um perigo real, como ameaça à integridade física ou psicológica da pessoa, o que leva a um estado emocional de alerta ante ao perigo. Já a ansiedade é um temor sem um objeto real, causada por lembranças e experiências anteriores, estando relacionados a uma etiologia multifatorial $^{4,6}$.

Experiências desagradáveis vividas anteriormente ou mesmo relatos de pessoas próximas fazem com que os pacientes temam os procedimentos que serão submetidos dentro do consultório odontológico, desconhecendo muitas vezes os recursos atuais para minimizar o estresse e a dor durante os tratamentos ${ }^{6}$. Os responsáveis/familiares possuem grande influência na introdução, aumento ou redução do medo e ansiedade dos pacientes, devido às suas próprias experiências, podendo assim favorecer ou desfavorecer o procedimento ${ }^{3}$.

A capacitação do profissional, associada à relação humanizada e acolhedora com os pacientes são fatores que contribuem para a minimização da ansiedade frente ao tratamento odontológico ${ }^{7}$. As faculdades de Odontologia têm promovido um bom acesso da população em relação à atenção em saúde bucal de forma gratuita e universal ${ }^{8}$.

Pacientes com diversos perfis $e$ necessidades de tratamentos odontológicos buscam atendimento contínuo nas clínicasescolas de Odontologia ${ }^{9}$. Esse tipo de atendimento deve responder à necessidade de formação e treinamento prático e técnico dos alunos, sem excluir o ideal ético de suprir as necessidades de saúde e as demandas dos pacientes que procuram o serviço, bem como a formação humanizada e ética dos profissionais de saúde ${ }^{10}$.

Diante da relevância de se conhecer os possíveis fatores que possam interferir no bom andamento do tratamento odontológico e na relação profissional-paciente, e, observando a grande quantidade de usuários que diariamente procuram atendimento na Clínica-Escola de Odontologia da Universidade Federal de Campina Grande (UFCG), o presente trabalho objetivou avaliar o perfil dos usuários e a prevalência e intensidade da odontalgia, ansiedade e medo relacionado ao tratamento odontológico desses pacientes.

MATERIAL E MÉTODO

Este trabalho foi aprovado pelo Comitê de Ética em Pesquisa com Seres Humanos do Hospital Universitário Alcides Carneiro da
Universidade Federal de Campina Grande/ HUAC - UFCG, sob protocolo no 3.021.299.

O estudo caracterizou-se como observacional e foi desenvolvido através da aplicação de três formulários semiestruturados e específicos. O Dental Fear Survey ${ }^{11}$, validado para a língua portuguesa por Cesar et al. ${ }^{12}$ (1993), para avaliação do medo ao tratamento odontológico, o de Avaliação da Dor de Dente ${ }^{13}$ e Modified Dental Anxiety Scale (MDAS) ${ }^{14}$ que avalia a ansiedade frente ao atendimento. As anotações foram realizadas por um pesquisador previamente calibrado, orientado a ser imparcial, e a obter as informações dos sujeitos sem induzir qualquer resposta. $O$ estudo realizou-se na Clínica-Escola de Odontologia da Universidade Federal de Campina Grande UFCG, localizada na cidade de Patos-PB a qual possui uma população estimada de 102.527 habitantes.

O universo foi composto por pacientes, maiores de 18 anos, que frequentaram a Clínica-Escola, durante o período de julho à setembro de 2018, e foram selecionados por conveniência na sala de espera, todos os dias da semana, de forma que abrangesse todas as especialidades. Foram incluídos todos os pacientes que aceitaram a participação na pesquisa por meio da assinatura do termo de consentimento livre e esclarecido e estavam devidamente cadastrados no serviço de atendimento da Clínica-Escola e excluídos os pacientes que apresentaram deficiências cognitivas e/ou neuropsicomotoras.

As respostas dos pacientes foram analisadas através de procedimentos de estatística descritiva realizada por meio do programa SPSS ${ }^{\circledR}$ (Statistical Package for the Social Sciences) versão 21.0 para Microsoft Windows, com teste de associações pela análise do Qui-quadrado, com nível de significância de $5 \%$, entre as variáveis da caracterização da amostra e a avaliação do medo, ansiedade e odontalgia. Os resultados foram expressos em números absolutos e percentuais, apresentados através de Tabelas.

RESULTADOS

\section{Análise Descritiva \\ - Perfil dos entrevistados}

Esta pesquisa foi realizada com 187 pacientes que procuraram a Clínica-Escola de Odontologia da Universidade Federal de Campina Grande, entre os períodos de julho a setembro de 2018. Quanto ao perfil dos entrevistados, $71,1 \% \quad(n=133)$ eram do sexo feminino e $28,9 \% \quad(n=54)$ do sexo masculino, com idade variando entre 18 e 75 anos e média 
de 49,1 anos, sendo que $33,7 \%(n=63)$ tinham entre 18-28 anos. A maior parte dos entrevistados $47,1 \% \quad(n=88)$ eram pardos, seguido por $46,5 \%(n=87)$ brancos e $6,4 \%$ $(n=12)$ negros.

Com relação à distribuição dos pacientes por Clínicas de Atendimento existentes na Clínica-Escola de Odontologia da Universidade Federal de Campina Grande-UFCG, pôde-se observar que Cirurgia foi a clínica com maior prevalência de procura pelos pacientes $(21,9 \%)$, seguido da Periodontia (20,3\%), Propedêutica $(19,3 \%)$, Dentística (18,2\%), Endodontia (10,7\%) e Prótese (9,6\%).

Com relação aos tipos de procedimentos a que os pacientes estavam sendo submetidos na Clínica-Escola no momento da entrevista, observou-se que a maior prevalência de atendimentos era de Restaurações (19,3\%), seguido de Exodontias (18,7\%), Raspagens (16,6\%), Consultas em geral $(13,9 \%)$, Cirurgias $(11,2 \%)$, Endodontia (10,7\%) e Prótese (9,6\%).

- Avaliação da dor dos pacientes atendidos na clínica-escola de Odontologia

Pôde-se observar que $85 \%(n=159)$ dos pacientes já sentiram "dor de dente" na vida, $32,1 \%(n=60)$ sentiram dor nos últimos 6 meses, a maior prevalência de duração em dias da dor foi entre 1 a 10 dias $(26,2 \% ; n=49)$ e durante 0 dia todo $(10,7 \% ; n=20)$ e a principal causa relatada foi o dente ter quebrado $(12,3 \%, n=23)$, sendo a palavra "Intolerante" como a que melhor descrevia a dor $(10,1 \% ; n=19)$. A Tabela 1 apresenta os dados relacionados a história de odontalgia dos pacientes entrevistados.

Tabela 1. Dados relacionados a queixa de odontalgia dos pacientes entrevistados. Patos - PB, Brasil - 2018.

\begin{tabular}{l|c|c}
\hline CARACTESRÍSTICAS DA DOR & $\mathbf{n}$ & $\mathbf{\%}$ \\
\hline Dor de dente & 159 & 85,0 \\
\hline Sim & 28 & 15,0 \\
\hline Não & 60 & 32,1 \\
\hline Dor últimos 6 meses & 127 & 67,9 \\
\hline Sim & 127 & 67,9 \\
\hline Não & 49 & 26,2 \\
\hline Duracão da dor em dias & 6 & 3,2 \\
\hline Sem dor & 4 & 2,1 \\
\hline O1-10 dias & 1 & 0,5 \\
\hline 21-30 dias & & \\
\hline Mais de 3o dias & 127 & 67,9 \\
\hline Duração da dor em horas por dia & 7 & 3,7 \\
\hline Sem dor & 15 & 8 \\
\hline Não lembra & 18 & 9,6 \\
\hline Menos de 1h & 20 & 10,7 \\
\hline Mais de 1h & & \\
\hline Odia todo & 127 & 67,9 \\
\hline Palavra que melhor descreve a dor & 12 & 6,4 \\
\hline Sem dor & 13 & 7,0 \\
\hline Leve & 13 & 7,0 \\
\hline Desconfortável & 3 & 1,6 \\
\hline Estressante & 19 & 10,1 \\
\hline Horrivel & & \\
\hline Intolerante & 127 & 67,9 \\
\hline Causa da dor & 8 & 4,3 \\
\hline Sem dor & 12 & 6,4 \\
\hline Não lembro & 11 & 5,9 \\
\hline Cavidade no dente & 6 & 3,2 \\
\hline Dora ao comer ou beber & 23 & 12,3 \\
\hline Durante o tratamento odontológico & \\
\hline Dente quebrado & & \\
\hline Fonte: Dados da pesquisa & & \\
\hline & & \\
\hline
\end{tabular}

- Avaliação da ansiedade relacionada ao tratamento odontológico

Pôde-se observar na Tabela 2 que $63,1 \%(n=118)$ relataram não sentir ansiedade por ir ao dentista, 55,1\% $(n=103)$ não sentem ansiedade na sala de espera, 65,8\% $(n=123)$ não sentem ansiedade pelo uso do motor, $73,3 \%(n=137)$ não relataram ansiedade ao uso dos instrumentais e 59,4\% $(n=111)$ não têm ansiedade à anestesia.

\section{- Avaliação do medo relacionado ao tratamento odontológico}

A Tabela 3 apresenta a avaliação do medo relacionado ao atendimento odontológico dos pacientes entrevistados. Observou-se que a maioria dos pacientes não demonstrou sentir medo em relação a fuga ao atendimento, as manifestações fisiológicas e ao medo provocado.

Tabela 2. Avaliação da ansiedade relacionada ao tratamento odontológico. Patos - PB, Brasil - 2018.

\begin{tabular}{|c|c|c|}
\hline CARACTERÍSTICAS DA ANSIEDADE DOS PACIENTES & $\mathbf{n}$ & $\%$ \\
\hline \multicolumn{3}{|l|}{$\begin{array}{l}\text { Ansiedade ao ir ao dentista } \\
\text { Nada ansioso }\end{array}$} \\
\hline $\begin{array}{l}\text { Un pouco ansioso } \\
\end{array}$ & & \\
\hline Muito ansioss & 228 & $\frac{15,0}{11,8}$ \\
\hline Bastante ansioso & 13 & 7,0 \\
\hline \multirow{2}{*}{\multicolumn{3}{|c|}{ Ansiedade na sala de espera }} \\
\hline & & \\
\hline $\begin{array}{l}\text { Nada ansioso } \\
\text { Um pouco ansioso }\end{array}$ & $\frac{103}{49}$ & $\frac{55,1}{26,2}$ \\
\hline Muito ansioso & 21 & 11,2 \\
\hline Bastante ansioso & 8 & 4,3 \\
\hline \multicolumn{2}{|l|}{ Ansiedade ao uso do motor } & 3,2 \\
\hline Nada ansioso & 123 & 65,8 \\
\hline Um pouco ansioso & 31 & 16,6 \\
\hline Muito ansioso & 16 & 8,6 \\
\hline Bastante ansioso & 8 & 4,3 \\
\hline \multirow{2}{*}{\multicolumn{3}{|c|}{ Ansiedade ao uso de instrumentais }} \\
\hline & 137 & \\
\hline Um pouco ansioso & 29 & 15,5 \\
\hline Muito ansioso & 11 & 5,9 \\
\hline $\begin{array}{l}\text { Bastante ansioso } \\
\text { Fxtremamente ensioso }\end{array}$ & 4 & 2,1 \\
\hline \multicolumn{3}{|l|}{ Ansiedade à anestesia } \\
\hline Nada ansioso & 111 & 59,4 \\
\hline Um pouco ansioso & 33 & 17,6 \\
\hline Muito ansioso & 20 & 10,7 \\
\hline $\begin{array}{l}\text { Bastante ansioso } \\
\text { Extremamente ansioso }\end{array}$ & 7 & 3,7 \\
\hline & & \\
\hline
\end{tabular}

Tabela 3. Avaliação do medo re
odontológico. Patos - PB, Brasil - 2018.

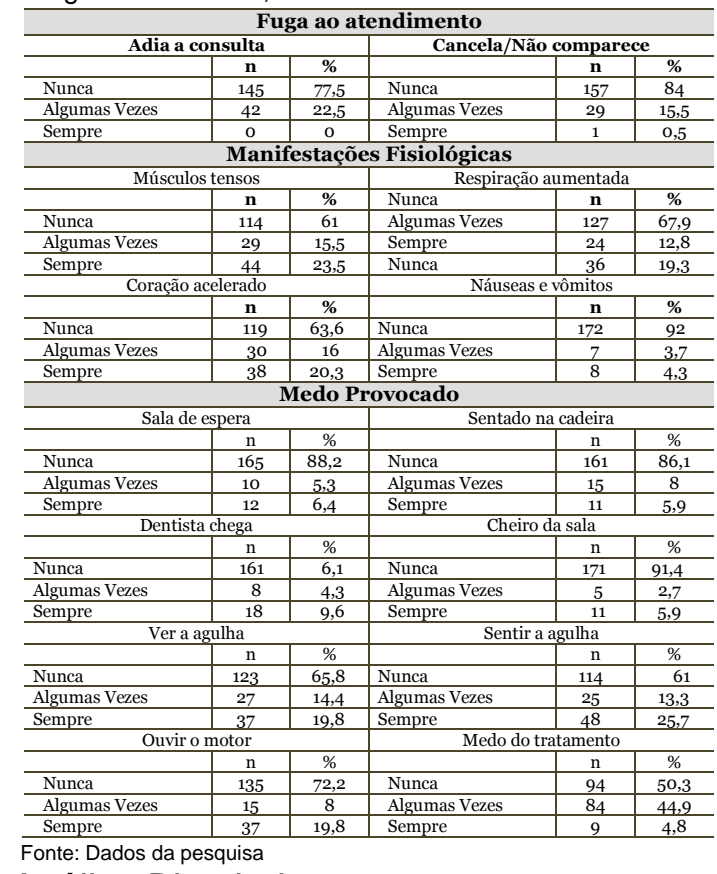

- Análise Bivariada

A Tabela 4 apresenta a avaliação da relação entre a odontalgia e as variáveis sexo e raça, onde observou-se relação estatisticamente 
significante entre odontalgia e raça, em ambas as situações analisadas $(p<0,05)$.

Tabela 4. Avaliação da relação entre a odontalgia e as variáveis sexo e raça. Patos - PB, Brasil - 2018.

\begin{tabular}{|c|c|c|c|c|}
\hline \multicolumn{5}{|c|}{ Dor de Dente na Vida } \\
\hline Variável & Não & Sim & Total & Valor de $\mathbf{p}^{(1)}$ \\
\hline \multicolumn{5}{|l|}{ Sexo } \\
\hline $\begin{array}{l}\text { Masculino } \\
\text { Feminino }\end{array}$ & $7(13,0)$ & $47(87,0)$ & $54(100,0)$ & 0,623 \\
\hline \multicolumn{5}{|l|}{ Raça } \\
\hline Branca & $20(23,0)$ & $67(77,0)$ & $87(100,0)$ & 0,016 \\
\hline & $\frac{7(8,0)}{1(8,3)}$ & $\frac{81(92,0)}{11(01,7)}$ & $\begin{array}{ll}88(100,0) \\
12(100,0)\end{array}$ & \\
\hline Negra & \multicolumn{4}{|c|}{ Dor de Dente nos Últimos 6 Meses } \\
\hline Variável & Ausente & Presente & Total & Valor de $\mathrm{p}^{(1)}$ \\
\hline & $\mathrm{n}(\%)$ & $\mathrm{n}(\%)$ & $\mathrm{n}(\%)$ & \\
\hline Sexo & & & & \\
\hline $\begin{array}{l}\text { Masculino } \\
\text { Feminino }\end{array}$ & $38(70,4)$ & $16(29,6)$ & $54(100,0)$ & \\
\hline $\begin{array}{l}\text { Feminino } \\
\text { Raca }\end{array}$ & & $44(33,1)$ & $133(100,0)$ & 0,647 \\
\hline Branca & $67(77,0)$ & & $87(1$ & $\mathbf{0 , 0 3 3}$ \\
\hline $\begin{array}{ll}\text { Parda } \\
\text { Negra }\end{array}$ & $54(61,4)$ & $34(38,6)$ & $88(100,0)$ & \\
\hline 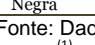 & $\begin{array}{l}\text { a (50,0) } \\
\text { a pesquisa }\end{array}$ & & ,o) & \\
\hline
\end{tabular}

A Tabela 5 apresenta a avaliação da relação entre as variáveis ansiedade e medo com o sexo e a raça. Observou-se dados estatisticamente significantes da ansiedade a anestesia em relação ao sexo $(p=0,022)$ e a raça $(p=0,013)$ e da ansiedade na sala de espera em relação ao sexo $(p=0,042)$. A avaliação do medo odontológico não apresentou relação estatisticamente significante com nenhuma das variáveis de perfil (sexo e raça).

Tabela 5. Avaliação da relação entre o medo e a ansiedade com o sexo e raça. Patos - PB, Brasil - 2018.

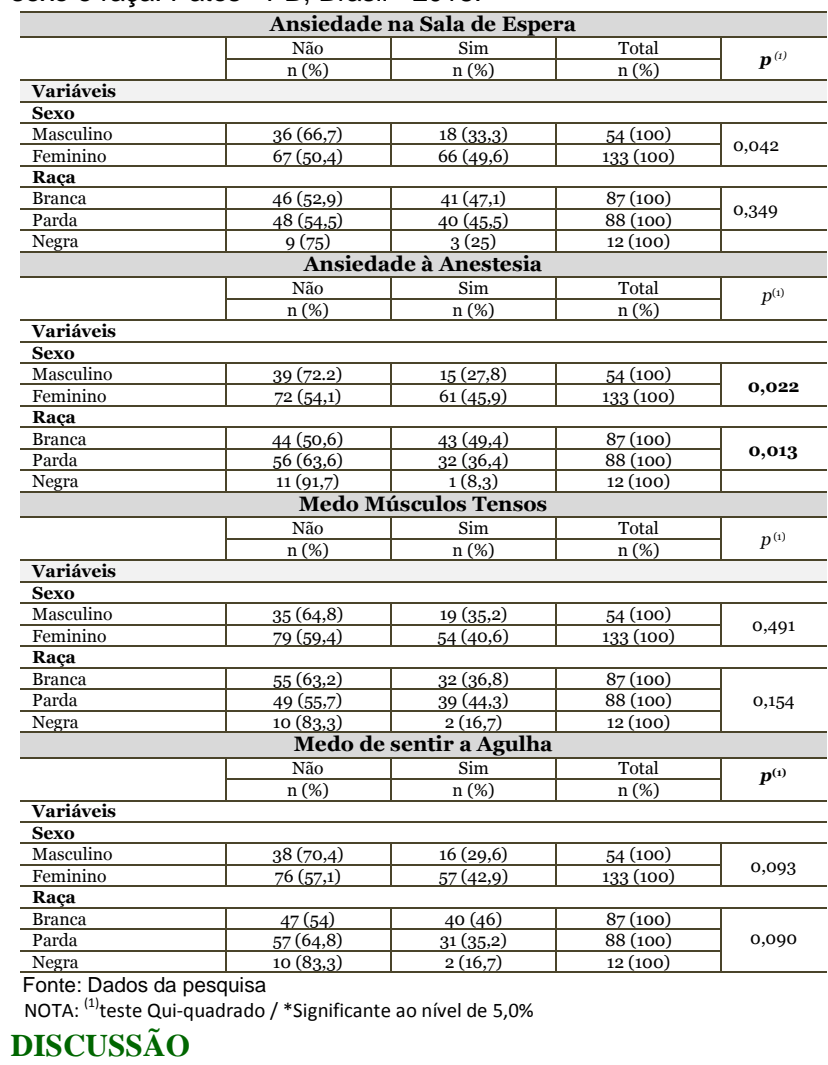

No presente estudo, observou-se uma expressiva maioria de pacientes do sexo feminino que procuraram 0 serviço de Odontologia da Clínica-Escola. Tal fato sugere que as mulheres se preocupam mais em cuidar da sua saúde do que os homens. O estudo se assemelhou aos dados encontrados por Domingos et al. ${ }^{15} \mathrm{e}$ Gonçalves et al. $^{16} \mathrm{e}$ contrariou o estudo de Oliveira et al. ${ }^{17}$ ao qual encontrou maior expressividade de pacientes do sexo masculino.

A clínica com maior prevalência de procura foi a clínica de cirurgia, tendo como o maior motivo a exodontia. Para Gonçalves et al. ${ }^{18}$ a clínica de cirurgia também foi a mais procurada para a realização de procedimentos. A demora dos pacientes em procurar o tratamento, por motivo de renda ou de falta de tempo, justificam o motivo de tantas exodontias, pois como Kanegane et al. ${ }^{19}$ observou, os pacientes só procuram tratamento quando a dor torna-se insuportável. Esses dados divergem do estudo de Murrer ${ }^{5}$ ao qual encontrou a maior prevalência de $41 \%$ na clínica de endodontia, tendo como principal motivo a odontalgia.

A odontalgia esteve presente em algum momento da vida da maioria dos entrevistados, entretanto observou-se que uma minoria declarou não ter sentido a mesma nos últimos 6 meses. Este fato está ligado a grande quantidade de pacientes se automedicando sem nenhum acompanhamento odontológico e que procuraram atendimento um longo tempo depois do fim da dor. A intensidade da dor intolerável foi a mais relatada, tendo duração do dia inteiro por até 10 dias o tempo da dor sentida pelos entrevistados. O estudo contrapõe-se ao trabalho de Ruivo et al. ${ }^{20}$ que, embora tenha obtido $54,75 \%$ de odontalgia ("dor de dente"), tem a intensidade da dor "intensa" como a mais relatada.

Quanto a avaliação da ansiedade, observou-se que a maioria dos entrevistados não relataram sentirem-se ansiosos em nenhuma das situações questionadas. Teles et al. ${ }^{21}$ também encontrou baixa prevalência de ansiedade ao ir ao dentista, na sala de espera, ao uso do motor, ao uso dos instrumentos e à anestesia, no entanto, Dantas et al. ${ }^{22}$ relataram que $70 \%$ dos pacientes declararam sentirem-se ansiosos diante de um procedimento odontológico.

Embora o estudo tenha encontrado ausência de ansiedade à anestesia, a mesma ainda representa desconforto aos pacientes, fato demonstrado quando da avaliação do medo de ver e sentir a agulha, que foram as situações com maior prevalência neste estudo, fato também observado Teles et al. ${ }^{21}$.

Conforme declarado por Queiroz et al. ${ }^{1}$ a ansiedade impacta na qualidade de vida, tendo como consequência a fuga dos pacientes aos tratamentos odontológicos ${ }^{23}$. A ansiedade altera 
a percepção da odontalgia gerando reações fisiológicas como transpiração excessiva, tremores, distúrbios gastrintestinais, aumento da frequência cardíaca, aumento da pressão arterial entre outras reações. Bottan et al. $^{24}$ declarou que essas sensações geram um ciclo vicioso, onde experiências passadas de dor provocam cada vez mais ansiedade e medo ao tratamento odontológico - fuga das consultas baixo nível de saúde bucal.

$O$ inciso citado anteriormente divergiu dos resultados da presente pesquisa, uma vez que embora tenha sido alta a prevalência de odontalgia dos entrevistados, os mesmos demonstraram pouca ansiedade e medo frente ao tratamento, onde pôde ser observada uma baixa prevalência de fuga ao atendimento e de manifestações fisiológicas.

O medo odontológico na infância, segundo Felix et al. ${ }^{25}$, ocorre devido a interações negativas com os profissionais e pacientes presentes no consultório ou ao manejo inadequado dos instrumentos, a presença da agulha e ao som dos instrumentos rotatórios. A maioria dos pacientes entrevistados relatou ausência de medo na sala de espera, ao cheiro da sala, ao dentista chegar, sentado na cadeira, ao ver agulha, ao sentir agulha, ao ouvir o motor e ao medo do tratamento, declarando que possuíam medo quando crianças, mas ao passar dos anos de vida foram perdendo o medo. Tal fato pode ser atribuído à humanização dos serviços de saúde e modernização dos equipamentos, tornando com o passar do tempo o atendimento menos traumático ${ }^{7}$.

A odontalgia ("dor de dente") apresentou relação estatisticamente significante com a raça, onde os brancos foram os que demonstraram menores índices de dor de dente na vida e nos últimos 6 meses. Tal fato corrobora com Oliveira et al. ${ }^{26}$ que observou que a população parda teve uma prevalência maior de odontalgia. Embora não tenha dado estatisticamente significante, observou-se ainda no presente estudo que a odontalgia teve maior prevalência pelo sexo feminino, similar ao trabalho de Murrer et al. ${ }^{5}$.

A ansiedade à anestesia e na sala de espera apresentaram relações estatisticamente significante com o sexo, constatando ser maior no gênero feminino, semelhante aos trabalhos de Murrer et al. ${ }^{5}$, Presoto et al. ${ }^{27}$, Oliveira et al. ${ }^{28}$ e Dantas et al. ${ }^{22}$ e se contrapõe ao de Teles et al. $^{21}$ que não encontrou diferenças entre os sexos. A ansiedade à anestesia também apresentou relação estatisticamente significante com a raça, onde os brancos mostraram-se mais ansiosos do que os pardos e negros. As mulheres também apresentam a maior prevalência de medo odontológico, embora o resultado não tenha dado estatisticamente significante.

Destaca-se que as raças pardas e brancas foram os maiores usuários dos procedimentos odontológicos disponíveis na Universidade Federal de Campina Grande, concordando com o estudo de Peres et al. ${ }^{29}$ onde está descrito que a população parda utiliza mais 0 acesso aos serviços odontológicos públicos de saúde.

CONCLUSÃO

Observou-se uma baixa prevalência de medo, ansiedade e dor de dente nos últimos 6 meses e uma alta prevalência de dor de dente sentida em algum momento da vida. Neste contexto, a maior prevalência de dor de dente foi em pacientes da raça parda, entretanto, a raça negra apresentou maior prevalência de dor de dente nos últimos 6 meses. Pacientes do sexo feminino e da raça branca demonstraram maior ansiedade ao tratamento odontológico.

\section{REFERÊNCIAS}

1. Queiroz MF, Verli FD, Marinho SA, Paiva PCP, Santos SMC, Soares JA. Dor, ansiedade e qualidade de vida relacionada à saúde bucal de pacientes atendidos no serviço de urgência odontológica. Cien Saude Colet. 2019;24(4): 1277-286.

2. Souza JGS, Martins AMEBL. Dor dentária e fatores associados em pré-escolares brasileiros. Rev Paul Pediatr. 2016;34(3): 336-42.

3. Felix LF, Brum SC, Barbosa CCN, Barbosa O. Aspectos que influenciam nas reações comportamentais de crianças em consultórios odontológicos. Rev Pró-UniverSUS. 2016;7(2): 13-6.

4. Medeiros LA, Ramiro FMS, Lima CAA, Souza LMA, Fortes TMV, Groppo FC. Avaliação do grau de ansiedade dos pacientes antes de cirurgias orais menores. Rev Odontol UNESP. 2013;42(5):357-63.

5. Murrer RD, Francisco SS, Endo MM. Ansiedade e medo no atendimento odontológico de urgência. ROBRAC. 2014; 23(67):196-201.

6. Martins RJ, Belila NM, Garbin CAS, Garbin AJI. Medo e ansiedade dos estudantes de diferentes classes sociais ao tratamento odontológico. Arch Health Invest.2017;6(1):43-7.

7. Mota LQ, Santos TA, Magalhães DBL. Humanização no atendimento odontológico: acolhimento da subjetividade dos pacientes atendidos por alunos de graduação nos campos de estágio. Rev bras ciênc Saúde. 2012;16(4):537-44. 
8. Pêgo SPB, Dias VO, Nascimento JE, Martelli Júnior $\mathrm{H}$, Martelli DRB, Santos $\mathrm{ML}$, et al. Avaliação dos serviços odontológicos prestados por acadêmicos: percepção do usuário. Rev Intercâmbio. 2016;7:138-47.

9. Matos PES, Mendes HJ, Santana ML. Acolhimento aos usuários do serviço de Odontologia de uma universidade pública. Rev ABENO. 2016;16(4):85-94.

10. Martelli Júnior $H$, Dias VO, Santos ML, Oliveira CA, Oliveira EC, Magalhães HTAT, et al. Dificuldades identificadas no atendimento odontológico após a triagem em uma universidade pública. Rev Intercâmbio. 2016; 7:274-83.

11. Kleinknecht RA, Keplac RK, Alexander LD. Origins and characteristics of fear of dentistry. J Am Dent Assoc. 1973;86(4):842-48.

12. Cesar J, Moraes AB, Milgrom $P$, Kleinknecht RA. Cross validation of a brazilian version of the dental fear survey. Community Dent Oral Epidemiol. 1993;21:148-50.

13. Goes PSA. The prevalence and impact of dental pain in Brazilian schoolchildren and their families [tese]. London: University College London; 2001.

14. Corah NL. Development of a dental anxiety scale. J Dent Res. 1969;48(4):596.

15. Domingos PAS, Rossato EM, Bellini A. Levantamento do perfil social, demográfico e econômico de pacientes atendidos na clínica de odontologia do centro universitário de Araraquara - Uniara. Rev Uniara 2014;17(1): 37-50.

16. Gonçalves CA, Vazquez FL, Ambrosano GMB, Mialhe FL, Pereira AC, Sarracini KLM et al. Estratégias para 0 enfrentamento do absenteísmo em consultas odontológicas nas Unidades de Saúde da Família de um município de grande porte: uma pesquisa-ação. Cien Saude Colet. 2015;20(2):449-60.

17. Oliveira ALP, Chagas SKM, Peixoto MOB, Peixoto FB, Cruz VSA, Ferreira SMS et al. Avaliação sociodemográfica e odontológica de pacientes com deficiência atendidos na clínicaescola de uma instituição de ensino superior. Rev ACBO. 2017;6(2):58-64.

18. Gonçalves ER, Verdi MIM. Os problemas éticos no atendimento a pacientes na clínica odontológica de ensino. Ciênc Saúde Colet. 2007;12(3):755-64.

19. Kanegane K, Penha SS, Borsatti MA, Rocha RG. Ansiedade ao tratamento odontológico em atendimento de urgência. Rev Saude Pública. 2003;37(6):786-92.

20. Ruivo MA, Alves MC, Bérzin MGR, Bérzin F. Prevalência de dor no segmento cefálico e sua associação com qualidade de vida na população geral do município de Piracicaba,
São Paulo: um estudo epidemiológico. Rev Dor. 2015;16(1):15-21.

21. Teles L, Cataldo D, Schneider LFJ, Cardoso M, Tannure PN. Baixo nível de ansiedade dos pacientes atendidos no curso de odontologia de uma instituição de ensino superior. Rev Odontol Univ Cid São Paulo. 2016;28(1):24-9.

22. Dantas DC, Marinho VL, Pinto KS. Prevalência de ansiedade frente à cirurgia oral: Um estudo com usuários da clínica escola de odontologia da Universidade de Gurupi. Rev Amazônia Sci Health. 2019;7(4):69-77.

23. Vermaire $\mathrm{JH}$, de Jongh $\mathrm{A}$, Aartman IH. Dental anxiety and quality of life: the effect of dental treatment. Community Dent Oral Epidemiol. 2008;36(5):409-16.

24. Bottan ER, Trentini L, Araújo SM. Ansiedade no tratamento odontológico: levantamento em estudantes do ensino fundamental do município de Pouso Redondo - SC. RFO UPF. 2007; 12(3):7-12.

25. Felix LF, Brum SC, Barbosa CCN, Barbosa O. Aspectos que influenciam nas reações comportamentais de crianças em consultórios odontológicos. Rev Pró-Uni. 2016;7(2):13-6.

26. Oliveira BA, Biazevic MGH, Michel-Crosato E. Prevalência de dor de dente, cárie dental e condições socioeconômicas: um estudo em adultos jovens brasileiros. Rev Odonto. 2011; 19(38):7-14.

27. Presoto CD, Cioffi SS, Dias TM, Loffredo LCM, Campos JADB. Escala de ansiedade odontológica: reprodutibilidade das respostas dadas em entrevistas telefônicas e pessoais. Rev. Pesq Bras Odontoped Clin Integr. 2011; 11(2):205-10.

28. Oliveira MLRS, Araújo SM, Bottan ER. Ansiedade ao tratamento odontológico: perfil de um grupo de adultos em situação não clínica. Rev Arq Ciên Saúde UNIPAR. 2015;19(3): 165-70.

29. Peres MA, Iser BPM, Boing AF, Yokota RTC, Malta DC, Peres KG. Inequalities in access to and utilization of dental care in Brazil: an analysis of the Telephone Survey Surveillance system for Risk and Protective Factors for Chronic Diseases (VIGITEL 2009). Cad Saúde Pública 2012; 28(supl.1):90-100. 


\section{CONFLITO DE INTERESSES}

Os autores declaram não haver conflitos de interesse

\section{AUTOR PARA CORRESPONDÊNCIA}

\section{Maria Luísa de Assis Braga}

Unidade Acadêmica de Ciências Biológicas,

Centro de Saúde e Tecnologia Rural,

UFCG - Universidade Federal de Campina Grande,

Rod. PB-110, 58708-110, Jatobá,

Patos - PB, Brasil.

Telefone: (83) 3511- 3045

E-mail:marialuisaabraga@hotmail.com 